\title{
Two Cases of Gastrocolocutaneous Fistula with a Long Asymptomatic Period after Percutaneous Endoscopic Gastrostomy
}

\author{
Hyo Sun Kim, Chang Seok Bang, Yeon Soo Kim, Oh Kyung Kwon, Min Sun Park, Jeong Ho Eom, \\ Gwang Ho Baik, Dong Joon Kim \\ Department of Internal Medicine, Hallym University College of Medicine, Chuncheon, Korea
}

Gastrocolocutaneous fistula is a rare complication of the percutaneous endoscopic gastrostomy (PEG) procedure. Typical symptoms usually occur in the first few months. We recently encountered 2 patients with 8 - and 33-month asymptomatic periods. A 74-year-old man presented with watery diarrhea for 1 month. He had undergone PEG 9 months earlier. During workup, an upper endoscopy and abdominal CT scan revealed the migration of the feeding tube into the transverse colon. He was discharged with a nasogastric tube after treatment. A 77-year-old man presented with sudden loosening of his PEG tube with a duration over 3 days. He had undergone PEG procedure three times until that time. During workup, a gastrocolocutaneous fistula was diagnosed. However, when previous studies were reviewed, an abdominal CT scan, which was done 6 months ago before the third PEG, showed the fistula already existed at that time, suggesting that it was created about 33 months earlier when he underwent the second PEG procedure. The patient died of pneumonia aggravation despite conservative treatment. Both a high index of suspicion and the careful inspection of the upper endoscopy are very important for early diagnosis regardless of symptoms. (Intest Res 2014;12:251-255)

Key Words: Percutaneous endoscopic gastrostomy; Gastrocolocutaneous fistula

\section{INTRODUCTION}

Percutaneous endoscopic gastrostomy (PEG) is commonly used for long-term enteral feeding for patients who are unable to swallow. PEG is usually considered a safe procedure associated with low mortality and a low rate of significant complications. ${ }^{1}$ Most complications of PEG are considered minor, including peristomal wound infection, tube dislodgement, and leakage. ${ }^{2,3}$ Gastrocolocutaneous fistula is a complication that develops from the perforation of a loop

Received August 20, 2013. Revised September 21, 2013.

Accepted September 28, 2013.

Correspondence to Yeon Soo Kim, Department of Internal Medicine, Hallym

University College of Medicine, Chuncheon Sacred Heart Hospital, 77 Sakju-

ro, Chuncheon 200-704, Korea. Tel: +82-33-240-5000, Fax: +82-33-255-

6244,E-mail: kimyeonsoo@hallym.or.kr

Financial support: None. Conflict of interest: None. of the colon, either by inadvertent puncture of the transverse colon during the PEG procedure or through erosion into the adjacent bowel over time. This complication is reported to be rare; a study by Pitsinis et al. ${ }^{4}$ revealed that the incidence rate of this complication was $0.5 \%$ in adults. Patients with this complication can remain without typical symptoms for a long time, and several cases with long asymptomatic periods have been reported. ${ }^{5-8}$ Therefore, awareness is helpful to avoid this complication, and a high index of suspicion can ensure an early diagnosis. Herein, we report 2 cases of iatrogenic gastrocolocutaneous fistula following PEG presenting after a long asymptomatic period.

๑ Copyright 2014. Korean Association for the Study of Intestinal Diseases. All rights reserved.

This is an Open Access article distributed under the terms of the Creative Commons Attribution Non-Commercial License (http://creativecommons.org/licenses/by-nc/3.0) which permits unrestricted non-commercial use, distribution, and reproduction in any medium, provided the original work is properly cited. 


\section{CASE REPORT}

\section{Case 1}

A 74-year-old man with a history of cerebral infarction was admitted with watery diarrhea that had lasted for 1 month. PEG had been performed 9 months prior to this admission. The patient's condition had been stable for 8 months after PEG. Vital signs and laboratory results upon admission were as follows: blood pressure, $80 / 40 \mathrm{mmHg}$; body temperature, $36.8^{\circ} \mathrm{C}$; white blood cell count, $18,300 / \mu \mathrm{L}$; hemoglobin level, $13.2 \mathrm{~g} / \mathrm{dL}$; BUN level, $10.3 \mathrm{mg} / \mathrm{dL}$; creatinine level, $0.9 \mathrm{mg} /$ dL; urine white blood cell count, high. His septic condition improved after adequate hydration and the administration of broad-spectrum antibiotics. However, the postprandial watery diarrhea persisted. On the 12th day of hospitalization, an upper endoscopy revealed a gastrocolic fistula and the internal bumper of the PEG tube was not seen (Fig. 1A). The feeding tube was withdrawn after the upper endoscopy.
On the same day, a colonoscopy also revealed a gastrocolic fistula in the transverse colon (Fig. 1B). Several metal clips were applied to seal the gastrocolic fistula opening (Fig. 1C). Abdominal CT performed before upper endoscopy showed that the bumper of the feeding tube had migrated into the colonic lumen (Fig. 2A) and revealed a fistula tract between the stomach and colon (Fig. 2B). In a radiologic study using gastrografin administered through the nasogastric tube 1 week later, there was no leakage of dye (Fig. 3). The patient was discharged with a nasogastric tube for feeding after the external opening of the gastrocolocutaneous fistula was closed. The patient has been alive in a nursing home for 3 months after discharge from the hospital.

\section{Case 2}

A 77-year-old man with a history of alcoholic dementia and intracerebral hemorrhage was referred by a nursing home to undergo an exchange of feeding tubes because
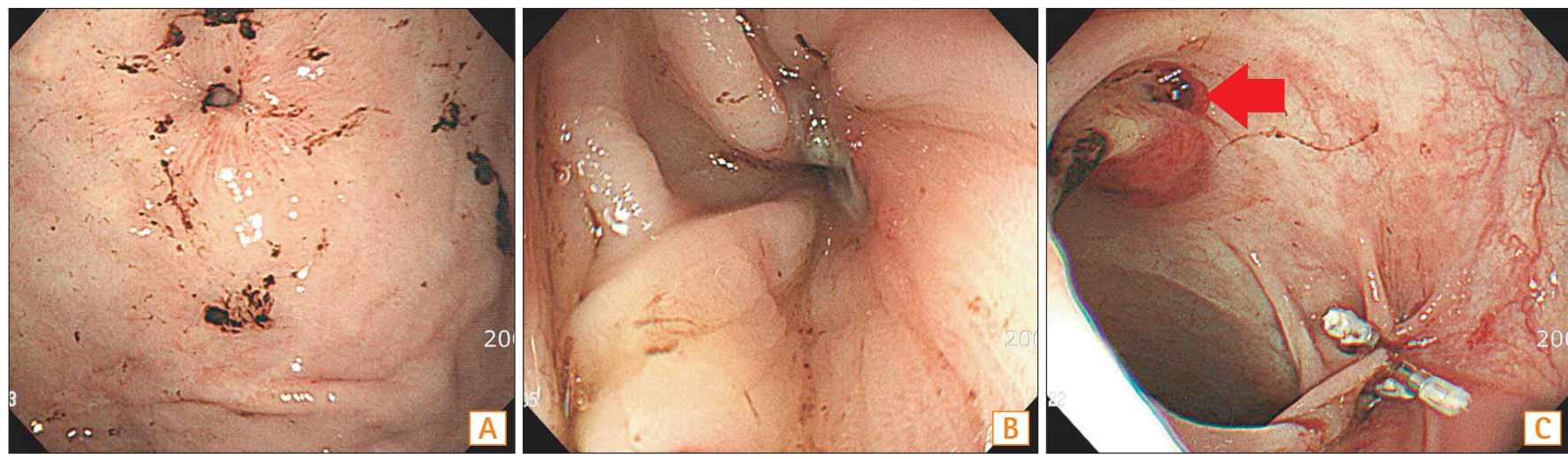

Fig. 1. Gastric and colonic view of fistula. (A) Upper endoscopy revealed a gastrocolic fistula and no visible bumper of the internal percutaneous endoscopic gastrostomy (PEG) tube. (B) Colonoscopy revealed a gastrocolic fistula in the transverse colon. (C) Three metal clips were successfully placed at the gastrocolic fistula opening during colonoscopy. The red arrow indicates the colocutaneous fistula opening, which remained under observation for spontaneous closure.
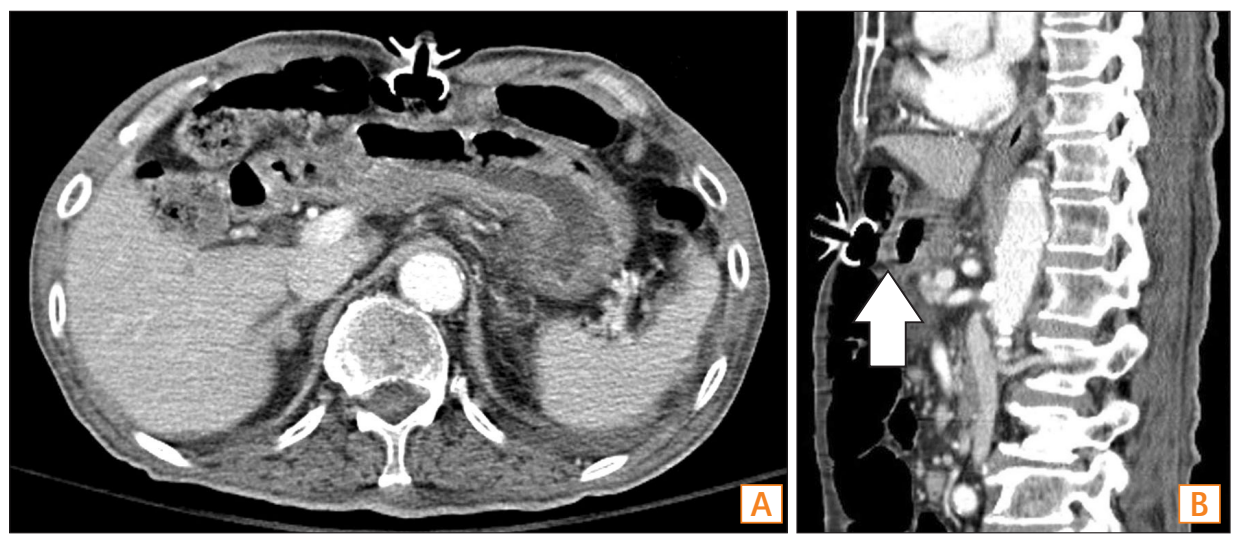

Fig. 2. Abdominal CT findings. (A) The bumper of the feeding tube that migrated into the colonic lumen (axial view). (B) The white arrow indicates the fistula's tract between the stomach and colon (sagittal view). 
his tube had suddenly loosened 3 days previously. He had undergone a PEG procedure 3 times and a simple exchange of PEG tubes twice. The first PEG procedure was performed

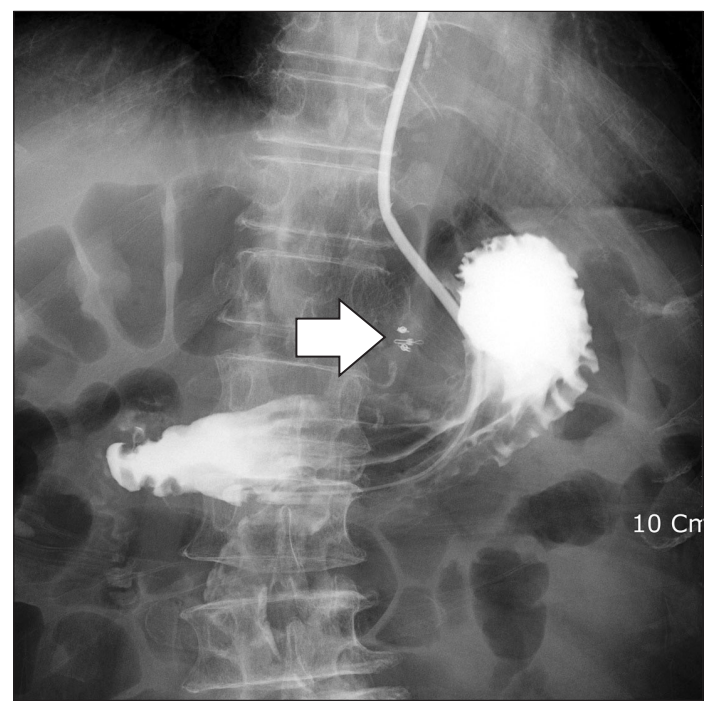

Fig. 3. Endoscopic sealing of the fistula. A radiologic study with gastrografin administered through a nasogastric tube was performed 1 week after the colonoscopic metal clipping. There was no leakage of dye from the stomach. The white arrow indicates the previous metal clips located at the colonic opening of the gastrocolic fistula.
33 months earlier for enteral feeding. The second PEG procedure was performed 2 weeks after the first because the patient had forcibly pulled out the initial feeding tube. The second PEG procedure had to be newly done because the tract of the first PEG site was obstructed shortly. The site of the second PEG was close by the first PEG site. The patient's condition was stable for 27 months after the second PEG procedure was done. The third PEG procedure was performed 6 months prior to this consultation owing to buriedbumper syndrome. At that time, the patient presented with a loosened tube and abdominal pain. Because an upper endoscopy revealed that the feeding tube bumper was buried in the gastric wall (Fig. 4A), the feeding tube was removed. The previous fistula tract required 4 days to close completely. Subsequently, a new gastrostomy was performed near the previous PEG site before discharge. The patient's condition had been stable for 6 months after the third PEG. Upon admission to our hospital, the patient's blood pressure was $90 / 60 \mathrm{mmHg}$ and body temperature was $36.1^{\circ} \mathrm{C}$. The blood test results were as follows: hemoglobin level, 3.7 $\mathrm{g} / \mathrm{dL}$; BUN level, $108.2 \mathrm{mg} / \mathrm{dL}$; creatinine level, $5.5 \mathrm{mg} / \mathrm{dL}$; estimated glomerular filtration rate, $10.7 \mathrm{~mL} / \mathrm{min} / 1.73 \mathrm{~m}^{2}$; and CRP, $229.99 \mathrm{mg} / \mathrm{dL}$. An upper endoscopy revealed that the bumper of the feeding tube was deeply buried within
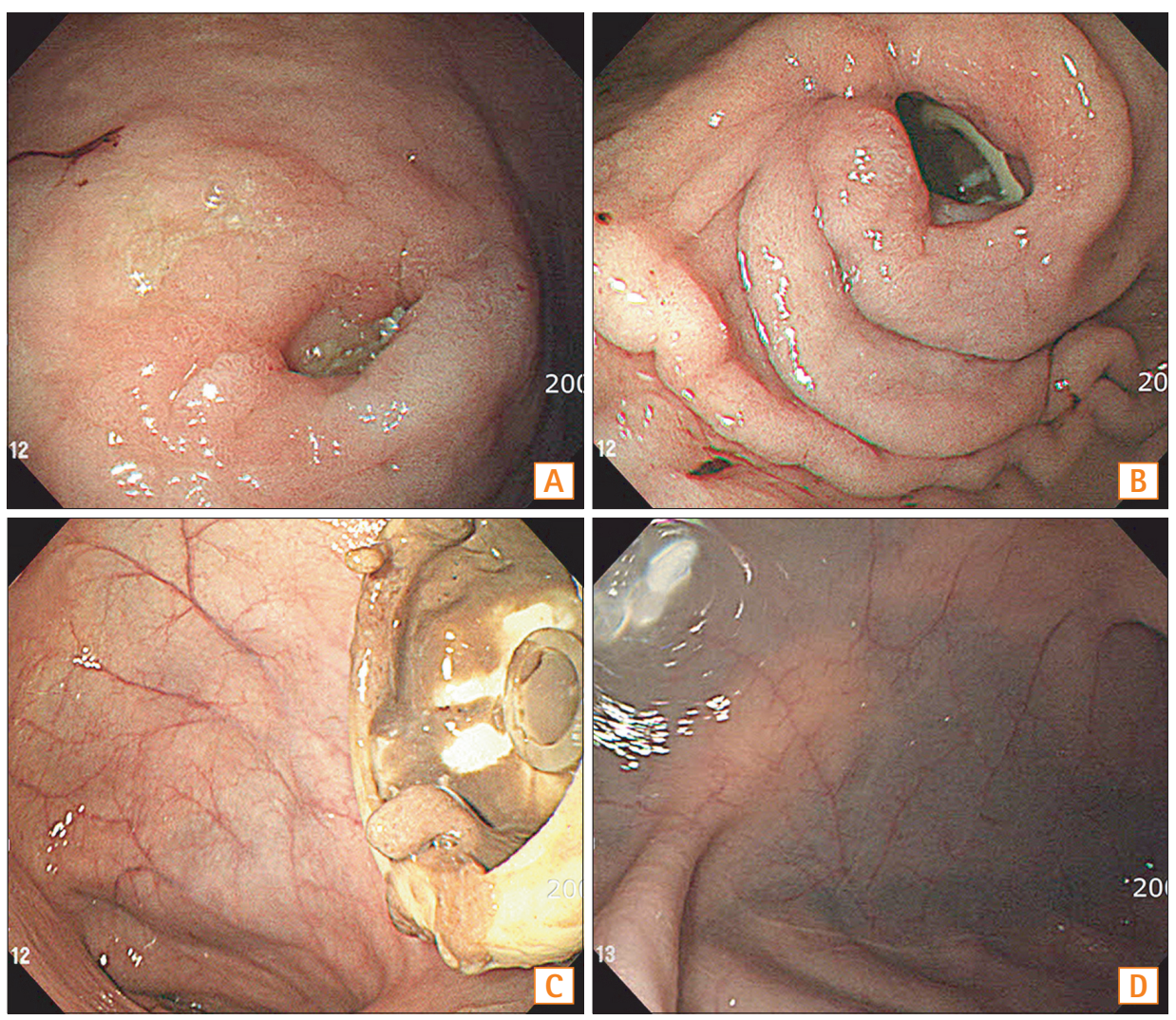

Fig. 4. Upper endoscopic findings. (A) An upper endoscopy which was done 6 months ago. The gastrocolocutaneous fistula was misdiagnosed as buried-bumper syndrome 6 months previously. (B) An upper endoscopy which was done at this time. The bumper of the feeding tube was deeply buried within the gastric wall and had formed a hole at the time of the patient's admission to our hospital. (C) The bumper with fecal material observed through the gastrocolic fistula. A large space was observed when the scope was advanced into the hole, and brownish material was attached to the bumper. (D) The colonic lumen observed through the gastrocolic fistula. The colonic lumen was identified by a bluish liver shadow and coIonic haustra, 3 weeks later. 
the gastric wall, forming a hole (Fig. 4B). A large space was observed when the scope was advanced into the hole, and brownish material was attached to the bumper (Fig. 4C). These findings suggested gastric wall perforation resulting from a deeply buried bumper. The feeding tube was exchanged to seal the gastric wall opening, and the bumper of the tube was positioned in the gastric cavity. The patient was admitted to the intensive care unit. Because peritonitis and aspiration pneumonia caused a septic condition with acute kidney injury, medical therapies including hydration, blood transfusion, continuous renal replacement therapy, and broad-spectrum antibiotics were applied. In consideration of the patient's condition, medical treatment, including total parenteral nutrition, was continued rather than using a surgical option. The feeding tube was withdrawn when pus discharged from the gastrostomy site on the 10th day of hospitalization. The patient's condition improved for a while, and the external opening of the colocutaneous fistula was nearly closed 3 weeks after admission. According to his family's request to insert a feeding tube through the gastrostomy tract, an upper endoscopy was performed again. The gastric wall opening was still unhealed. When the scope was advanced into the opening, the lumen of the transverse colon and a bluish liver shadow were identified (Fig. 4D). The diagnosis of a gastrocolocutaneous fistula was confirmed at that time. The patient died of multiple organ failure caused by pneumonia aggravation 1 week later.

\section{DISCUSSION}

The exact mechanism of gastrocolocutaneous fistula is not well known. However, the most plausible theory is the interposition of the colon, usually the splenic flexure, between the anterior abdominal wall and the gastric wall. ${ }^{9,10}$ Conditions such as adhesion from a previous laparotomy or rotation of the stomach from insufflated air via endoscopy can juxtapose the colon with the anterior abdominal wall. ${ }^{11}$ The condition of gastrocolocutaneous fistula is divided into 3 stages according to the position of the bumper (Fig. 5). In the intervening period, the transmural migration of the feeding tube shows endoscopic findings that are similar to buriedbumper syndrome (Fig. 5B). Ultimately, the PEG bumper migrates into the intracolonic space through the gastrocolic fistula (Fig. 5C).

The leakage of gastric contents through the gastrocolic fistula into the colon leads to typical symptoms such as presence of diarrhea that contains food. The reverse condition causes feculent vomiting and the appearance of fecal material. ${ }^{9,10,12}$ In cases in which the fistula is completely sealed by the tube, these symptoms are delayed until the seal loosens. ${ }^{7}$ In cases of intracolonic migration, characteristic symptoms such as the sudden onset of diarrhea and cramping immediately after tube feeding, an odorous fecal exudate from the stoma, and loosening of the tube appear. ${ }^{6}$ In addition to these symptoms, a long bowel preparation period, which was approximately 1 week in Case 1, may be associated with the intracolonic migration of the bumper of the feeding tube.

Techniques using both transillumination and finger pressure as a guide to place the puncture site are useful for preventing this complication. Guidance by ultrasound or CT can be used selectively but may have limited benefit. A study by Foutch et al. ${ }^{12}$ suggested that an aspirating syringe filled

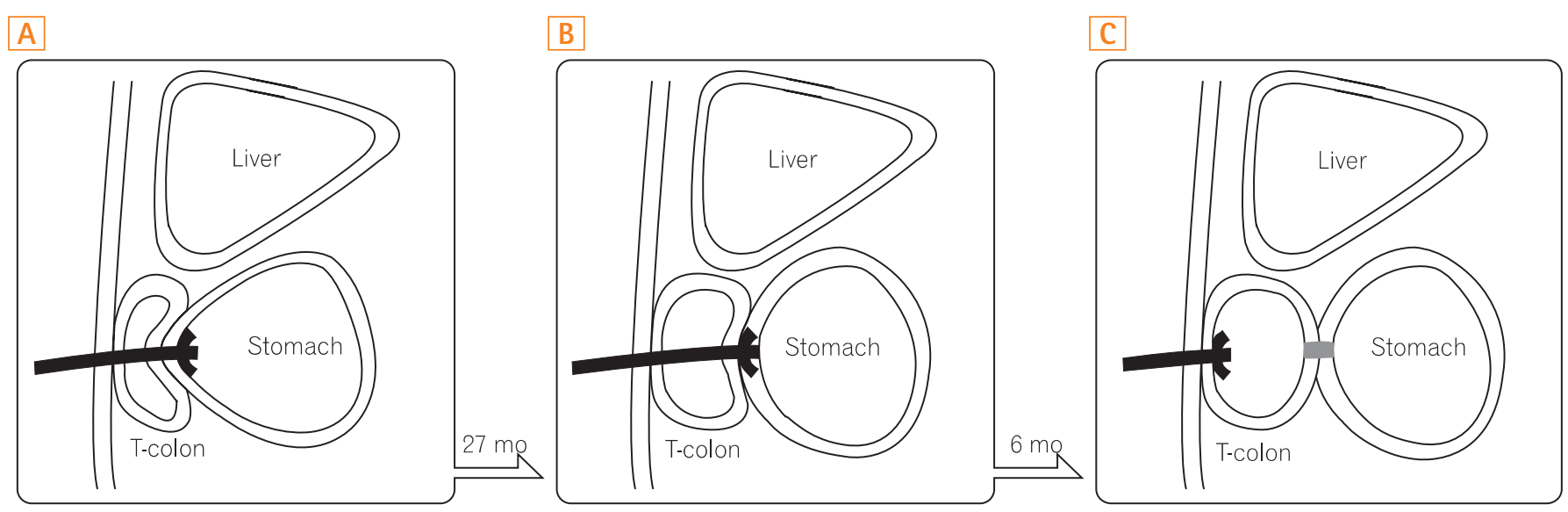

Fig. 5. Schematic diagrams of the 3 stages based on the position of the bumper in Case 2. (A) The gastrocolocutaneous fistula was created during the percutaneous endoscopic gastrostomy (PEG) placement, and the transverse colon was pressed tightly between the stomach and the abdominal wall (the first stage). (B) During the intervening period, the transmural migration of the feeding tube showed endoscopic findings similar to those of buriedbumper syndrome (the second stage). (C) The bumper migrated into the intracolonic space through the gastrocolic fistula (the third stage). 
with saline could be used to identify the intervening colon between the skin and the stomach if air bubbles appeared in the syringe prior to the endoscopic visualization of the needle in the gastric lumen.

This complication can be fatal if it is not detected early and managed properly. ${ }^{13,14}$ Upper endoscopy is recommended for early diagnosis in patients with typical symptoms, such as diarrhea containing food, fecal material in the feeding tube, feculent vomiting, or a loosened tube. Thereafter, contrast radiography with a water-soluble contrast medium, such as gastrografin, ${ }^{15}$ administered through the feeding tube or abdominal CT may help confirm the diagnosis in patients with typical endoscopic findings, such as fecal material attached to the PEG bumper, a bluish shadow, colonic haustra, or an invisible internal PEG tube bumper.

Historically, the key treatment for gastrocolocutaneous fistula was to allow the fistula to close after the feeding tube was removed. Recently, endoscopic treatment of fistulas has been attempted, although surgery is often required ${ }^{16-18}$ In our first case, the gastrocolic fistula was successfully sealed using metal clips during colonoscopy, and the colocutaneous fistula spontaneously closed.

In summary, the 3 key take-home messages from our cases are as follows. First, some techniques (such as transillumination, aspirating syringe usage, and application of a small amount of insufflated air) are helpful for preventing fistulas. Second, a careful inspection of the upper endoscopy in patients with typical symptoms is useful for early diagnosis. Finally, if typical upper endoscopic findings, such as fecal material attached to the PEG bumper, a bluish shadow, colonic haustra, or buried bumper, are observed, the possibility of a gastrocolocutaneous fistula must be considered.

\section{REFERENCES}

1. Berger SA, Zarling EJ. Colocutaneous fistula following migration of PEG tube. Gastrointest Endosc 1991;37:86-88.

2. Blomberg J, Lagergren J, Martin L, Mattsson F, Lagergren P. Complications after percutaneous endoscopic gastrostomy in a prospective study. Scand J Gastroenterol 2012;47:737-742.

3. Croaker GD, Najmaldin AS. Laparoscopically assisted percutaneous endoscopic gastrostomy. Pediatr Surg Int 1997;12:130131.

4. Pitsinis V, Roberts P. Gastrocolic fistula as a complication of percutaneous endoscopic gastrostomy. Eur J Clin Nutr 2003;57: 876-878.
5. Huang SY, Levine MS, Raper SE. Gastrocolic fistula with migration of feeding tube into transverse colon as a complication of percutaneous endoscopic gastrostomy. AJR Am J Roentgenol 2005;184:S65-S66.

6. Lenzen H, Weismuller T, Bredt M, Bahr M. Education and imaging. Gastrointestinal: PEG feeding tube migration into the colon; a late manifestation. J Gastroenterol Hepatol 2012;27:1254.

7. Marcy PY, Magne N, Lacroix J, Bailet C. Late presentation of a gastrocolic fistula after percutaneous fluoroscopic gastrostomy. JBR-BTR 2004;87:17-20.

8. Naehrlich L, Carbon R, Lang T, Behrens R. Two rare complications of percutaneous endoscopic gastrostomy: obstruction of the pylorus and gastrocolic fistula occurring in one patient. Klin Padiatr 2001;213:329-331.

9. Minocha A, Rupp TH, Jaggers TL, Rahal PS. Silent colo-gastrocutaneous fistula as a complication of percutaneous endoscopic gastrostomy. Am J Gastroenterol 1994;89:2243-2244.

10. Payne KM, King TM, Eisenach JB. The technique of percutaneous endoscopic gastrostomy. A safe and cost-effective alternative to operative gastrostomy. J Crit Illn 1991;6:611-619.

11. McClave SA, Chang WK. Complications of enteral access. Gastrointest Endosc 2003;58:739-751.

12. Foutch PG, Talbert GA, Waring JP, Sanowski RA. Percutaneous endoscopic gastrostomy in patients with prior abdominal surgery: virtues of the safe tract. Am J Gastroenterol 1988;83:147150.

13. Holder TM, Leape LL, Ashcraft KW. Gastrostomy: its use and dangers in pediatric patients. N Engl J Med 1972;286:1345-1347.

14. Joo YJ, Koo JH, Song SH. Gastrocolic fistula as a cause of persistent diarrhea in a patient with a gastrostomy tube. Arch Phys Med Rehabil 2010;91:1790-1792.

15. Okutani D, Kotani K, Makihara S. A case of gastrocolocutaneous fistula as a complication of percutaneous endoscopic gastrostomy. Acta Med Okayama 2008;62:135-138.

16. Hwang JH, Kim HW, Kang DH, et al. A case of endoscopic treatment for gastrocolocutaneous fistula as a complication of percutaneous endoscopic gastrostomy. Clin Endosc 2012;45:9598.

17. Lynch CR, Fang JC. Prevention and management of complications of percutaneous endoscopic gastrostomy (PEG) tubes. Practical Gastroenterology 2004;28:66-77.

18. Melmed GY, Kar S, Geft I, Lo SK. A new method for endoscopic closure of gastrocolonic fistula: novel application of a cardiac septal defect closure device (with video). Gastrointest Endosc 2009; 70:542-545. 